\title{
Research on high-order flux reconstruction method with adaptive mesh for shock capturing
}

\section{Xiuqiang Ma}

Nanjing University of Aeronautics and Astronautics College of Aerospace Engineering

Jian Xia ( $\boldsymbol{D}$ jxia@nuaa.edu.cn )

Nanjing University of Aeronautics and Astronautics College of Aerospace Engineering https://orcid.org/0000-0003-3182-1461

Hao Fu

Nanjing University of Aeronautics and Astronautics College of Aerospace Engineering

Research

Keywords: Flux Reconstruction, Shock capturing, Artificial Diffusivity, In-cell Piecewise Integrated Solution, Adaptive Mesh Refinement

Posted Date: May 29th, 2020

DOI: https://doi.org/10.21203/rs.3.rs-31697/v1

License: (1) (1) This work is licensed under a Creative Commons Attribution 4.0 International License. Read Full License 
Xiuqiang Ma1, Jian $\mathrm{Xia}_{1}^{*}$ and Hao Fu1

\begin{abstract}
High order schemes have been developed for a quite long time, and many famous schemes arise like Discontinuous Galerkin (DG), Spectral Difference (SD) and WENO schemes, etc. The Flux Reconstruction (FR) scheme proposed by Huynh has attracted the attention of researchers for its simplicity and efficiency. It's written in differential form and bridges the DG and SD schemes, which can be constructed with a proper choice of parameter. In this paper, realize FR scheme based on the framework of the open source Adaptive Mesh Refinement (AMR) library p4est. To capture shock sharply, the performance of Localized Laplacian Artificial Viscosity (LLAV) and In-cell Piecewise Integrated Solution methods are compared. As an important way of reducing computational cost, AMR technique integrated in p4est is also combined with FR. The performance of the developed code is tested in both one dimensional and two dimensional and get some quite attracting results.
\end{abstract}

Keywords: Flux Reconstruction, Shock capturing, Artificial Diffusivity, In-cell Piecewise Integrated Solution, Adaptive Mesh Refinement

\title{
1 Introduction
}

There has been a great development in computational fluid dynamics in recent years. The Finite Volume Method (FVM) has obtained a great success, and was applied in both commercial application and academic research widely. However with the increasing requirement for efficiency and precision, FVM shows a poor performance in the simulation of turbulence, capturing of shock, combustion, or the vortex propagation. This motivates researchers to explore new methods to overcome this difficulty.

There are basically two ways to minimize error. One is the development of high order schemes. High order methods show a better performance than FVM by constructing high order distribution function with local information. The well-known high order scheme Discontinuous Galerkin method (DG) was first proposed by Reed [1] and was extended to CFD by Shu [2] later. It's easy to implement and is stable, which is important in CFD research. It has attracted researches to modify DG scheme. Later based on DG, many mutants like the SD [3], SV [4] [5] schemes of DG were developed.

In 2007 Huynh [6] proposed Flux Reconstruction scheme. It is easy to implement and with a proper choice of solution points and correction function, both DG scheme and SD scheme can be constructed directly, and is simpler than both of them in some way. Because FR scheme only involves local cells and the values on the boundary of adjacent cells, which makes the scheme

*Correspondence: jxia@nuaa.edu.cn

${ }_{1}$ College of Aerospace Engineering, Nanjing University of Aeronautics and Astronautics, Nanjing, 210016, China 
compact and suitable for parallel simulation.

In 2009, Wang developed the LCP (Lifting Collocation Penalty) [7] scheme, which adds a correction part in control equations, and can be regarded as an extension of FR scheme to triangular elements. Later LCP was extended to Navier-Stokes equations in both three dimensional and two dimensional [8][9][10]. As FR and LCP scheme have much in common, Wang and Huynh called these two schemes CPR (Correction Procedure with Reconstruction) scheme. Jameson [11] first proved that SD scheme is stable with Soblev norm. Later based on Jameson's criteria a new scheme called ESFR [12] (energy stable flux reconstruction) which is a one parameter scheme was proposed by Vincent et al. With a parameter "c" of infinite range chosen, DG scheme, SD scheme or the g2 scheme in Huynh's paper can be constructed easily. With the help of math tools, Jameson [13] optimized the FR and ESFR approach and gave the optimized versions which showed a better performance than the original ones.

To reduce the calculation amount of FR approach, DFR (Direct Flux Reconstruction) scheme [14] was developed, which doesn't need to calculate correction part and is also suitable for matrix operation which is more efficient. In 2016 Romeo [15] extended DFR to triangular element, which adds an imaginary edge to triangle cell and gets a quadrant. In this way the DFR scheme can be implemented in unstructured mesh without much change in the primary programs and is easy to realize. Later Romeo and Witherden [16] extended DFR to triangular elements in advectiondiffusion problem. In 2018 Wang Lai [17] proposed the CDFR (Compact Direct Flux Reconstruction) scheme, it doesn't need to construct high order polynomials explicitly, and is proven equivalent with DFR.

It's easy for high order schemes to get high accuracy when solutions are smooth. However when there exists shock, contact discontinuities or vacuum, non-physical oscillation could occur near these discontinuities, which could cause fake solutions or even divergence. To overcome this problem, a lot of researches aiming at suppressing oscillations near discontinuities have been done. The limiter and artificial viscosity are the most popular techniques. Basically the limiter tends to limit the solution to a specified range which is applied directly to solution rather than flux. In DG type schemes Cockburn [18] proposed a TVB type limiter, Du Jie [19] developed a WENO type limiter for CPR scheme. Park et al [20] introduced a multidimensional limiter for CPR. And there are other limiters can be found in [21] [22]. Artificial viscosity mainly adds a viscosity term in control equations, equivalent to apply a viscous term in the whole domain. In artificial viscosity, the LAD (Localized Artificial Diffusivity) [23] and LLAV (Localized Laplacian Artificial Viscosity) [24] and have been applied widely [25, 26, 27, 28]. And there are some other ways of shock capturing like filtering technique can be found in [29] and the newly developed In-cell Piecewise Integrated Solution method [30][31].

The other way to minimize error is using smaller computing cells. And compared to simply refining grids the Adaptive Mesh Refinement (AMR) technique is a clear choice which can get more precise results with much less computational cost. And has been widely used in many kinds of commercial software.

AMR changes grid structure according to flow properties to refine grids where solution changes rapidly, or coarsen grids otherwise. Basically there are three types of AMR [32][33], the adaptive $\mathrm{h}-, \mathrm{r}$ - and p-refinement methods. Adaptive h-refinement mainly refines or coarsens cells which would change the structure of primary grid, makes it more complicate. Adaptive r-refinement stands for moving grid points without changing of grid connecting relation. Besides, because of 
moving grid points, adaptive r-refinement could cause poor quality of cells. The p-refinement is to adaptively use different orders of solution. Also there are some researches that combine these methods.

It's obvious to utilize the advantages of both high order scheme and AMR. In this paper combine FR scheme with AMR technique and test the shock capturing ability of LLAV and In-cell Piecewise Integrated solution methods. The remainder of this paper is organized as follow. The FR scheme is introduced in Sec.2. Different shock capturing methods and AMR technique are introduced in Sec.3. Sec.4 shows some numerical results in both one and two dimensional. The research is summarized briefly in final section.

\section{FR scheme}

\subsection{One dimensional FR scheme}

In this section FR scheme is introduced in one dimensional conservation law

$$
\frac{\partial u}{\partial t}+\frac{\partial f\left(u, u_{x}\right)}{\partial x}=0 \text {. }
$$

First consider partitioning the computational domain $[\mathrm{a}, \mathrm{b}]$ into $N$ non-overlapping cells, define i-th cell with $E_{i}=\left[x_{i-1 / 2}, x_{i+1 / 2}\right], i \in 1,2 \ldots \ldots . N$. In order to normalize FR scheme, transform each cell into a standard cell $[-1,1]$, and the local coordinate is $\xi$, the transformation between $x$ and $\xi$ is

$$
\begin{aligned}
& x(\xi)=x_{i}+\xi h_{i} / 2 \\
& \xi(x)=2\left(x-x_{i}\right) / h_{i}
\end{aligned},
$$

here $h_{i}=x_{i+1 / 2}-x_{i-1 / 2}$ is the length of $\mathrm{i}$-th cell, $x_{i}=\left(x_{i+1 / 2}+x_{i-1 / 2}\right) / 2$ is the coordinate of the center of i-th cell. Here choose K points $\xi_{k}, k=1,2 \ldots . . . K$ as solution points to define the standard cell, the left and right boundary points are flux points. Assume the value $u_{i, k}\left(t^{n}\right)$ at time $t$ on each solution point is known, without confusion write $u_{i, k}\left(t^{n}\right)$ as $u_{i, k}(t)$. In FR, there are many kinds of solution points to use, such as Gauss points, Lobatto points and equidistant points. With Lagrange interpolation, a polynomial of degree K-1 can be constructed.

$$
\begin{aligned}
& u_{i}(\xi)=\sum_{k=1}^{K} u_{i, k} \phi_{k}(\xi) \\
& f_{i}(\xi)=\sum_{k=1}^{K} f_{i, k} \phi_{k}(\xi), \\
& f_{i, k}=f\left(u_{i, k}\right)
\end{aligned}
$$

where $\phi_{k}$ is the K-th Lagrange basis function

$$
\phi_{k}(\xi)=\prod_{j=1, j \neq k}^{K} \frac{\xi-\xi_{j}}{\xi_{k}-\xi_{j}}
$$

It's easy to know that the solutions on the boundary of adjacent cells are basically different. To construct a continuous distribution function, Huynh introduced the correction concept which adds a correction function of degree $\mathrm{K}$ to flux function.

To consider the interaction of neighboring cells, common value on boundary needs to be considered. Use $L$ and $R$ to represent the value on left and right boundary,

$$
u_{L}=u_{i+1 / 2, L}=u_{i}(1), \quad u_{R}=u_{i+1 / 2, R}=u_{i+1}(-1),
$$


Here the flux function is not continuous. Riemann flux id used as the common flux, in this paper the well-known Roe scheme is chosen.

$$
f_{c}^{c o m}=f_{R o e}\left(u_{L}, u_{R}\right) .
$$

With correction function $g(\xi)$, the continuous flux function can be written as

$$
f_{i}^{\delta C}(\xi)=f(\xi)+\left(f_{i-1 / 2}^{c o m}-f_{i}(-1)\right) g_{L}(\xi)+\left(f_{i+1 / 2}^{c o m}-f_{i}(1)\right) g_{R}(\xi),
$$

here the last two terms are the correction of left and right boundary. $g_{L}$ and $g_{R}$ are the left and right correction function respectively, which satisfy

$$
\begin{array}{ll}
g_{L}(-1)=1, & g_{L}(1)=0 \\
g_{R}(-1)=0, & g_{R}(1)=1
\end{array} .
$$

There are many types of correction function can be applied which can be found in Huynh's research. In particular, Radau function makes the scheme of order $2 \mathrm{~K}-1$. In this paper, Radau function is applied to get high accuracy.

To solve the conservation law, flux function needs to be differentiated.

$$
\left(f_{i}^{\delta C}\right)_{\xi}\left(\xi_{k}\right)=\left(f_{i}\right)_{\xi}\left(\xi_{k}\right)+\left(f_{i-1 / 2}^{c o m}-f_{i}(-1)\right) g_{L}^{\prime}\left(\xi_{k}\right)+\left(f_{i+1 / 2}^{c o m}-f_{i}(1)\right) g_{R}^{\prime}\left(\xi_{k}\right) \text {. }
$$

By now the derivative of flux in computational domain is complete, with the transform equation of $\mathrm{x}$ and $\xi$, we can get the derivative in physical domain

$$
\left(f_{i}^{\delta C}\right)_{x}\left(x_{i, k}\right)=\frac{2}{h_{i}}\left(f_{i}^{\delta C}\right)_{\xi}\left(\xi_{k}\right) \text {. }
$$

And the conservation law becomes

$$
\frac{d u_{i, k}}{d t}=-\frac{2}{h_{i}}\left(f_{i}^{\delta C}\right)_{\xi}\left(\xi_{k}\right) \text {. }
$$

Huynh pointed that in linear cases the choice of correction function has a great influence on the stability and precision, the order of scheme is irrelevant with the type of solution points. In Jameson's research [34], the type of solution point is important for it can affect the energy property and aliasing error when conservation laws are non-linear. Gauss points can eliminate aliasing error. In this paper Gauss points are chosen as solution points. Finally, explicit or implicit time marching methods can be applied to solve the differential equations.

\subsection{Two dimensional FR scheme}

For quadrilateral mesh, it's easy to extend FR scheme to two dimensional without much effort. In this paper the grid lines of applied mesh are all horizontal or vertical. And the calculation can be conducted directly in one dimensional manner.

\section{Shock Capturing and Adaptive Mesh Refinement}

To overcome the problem of high order schemes in shock cases, shock capturing techniques need to be applied. In this section the artificial viscosity and in-cell piecewise integrated solution methods are introduced and compared. To improve computing efficiency the adaptive mesh refinement technique is also presented. 


\subsection{Artificial Viscosity}

Basically Artificial Viscosity technique is constructed based on local value, which is easy to implement in high order schemes. But how to define the amount of viscosity is still an open question for there are adjustable parameters that are problem dependent.

In this paper, the LLAV (Localized Laplacian Artificial viscosity) method proposed by Persson [24] is applied, which has the property of limiting shock within a cell. The LLAV method adds a Laplacian diffusivity term in control equations, which makes the equations become

$$
\frac{\partial \mathbf{U}}{\partial t}+\nabla \cdot \mathbf{F}(\mathbf{U})=\nabla \cdot(\varepsilon \nabla \mathbf{U})
$$

On the right hand side is the Laplacian artificial viscosity term, $\varepsilon$ is the viscosity parameter, which is relevant to the property of local flow. In Persson's research, $\varepsilon \square h / p, h$ is the cell size, $P$ is the order of solution polynomial. And $\varepsilon$ is expressed as follow

$$
\varepsilon= \begin{cases}0 & s_{e}<s_{0}-\kappa \\ \frac{\varepsilon_{0}}{2}\left(1+\sin \frac{\pi\left(s_{e}-s_{0}\right)}{2 \kappa}\right) & s_{0}-\kappa \leq s_{e} \leq s_{0}+\kappa, \\ \varepsilon_{0} & s_{e}>s_{0}+\kappa\end{cases}
$$

here $s_{e}$ is the smooth indicator which will be introduced shortly. In equation(13), $\varepsilon_{0}$ is the added artificial viscosity which can be regarded as the function of $\Delta \xi_{\max }$ and $P e^{\prime}$ clet number $P e$, size of grid cell $h$ and the maximum characteristic speed $|\lambda|_{\max }$

$$
\varepsilon_{0}=f\left(\Delta \xi_{\max }, P e\right) \cdot h \cdot|\lambda|_{\max },
$$

where $\Delta \xi_{\max }$ is the ratio of the distance of two solution points and cell length, which has a range $\Delta \xi_{\max } \in[0,1]$. Set $P e$ equals to 2 . And there are different functions for function $f\left(\Delta \xi_{\max }, P e\right)$ as shown in Fig. 1. In this paper linear function is chosen.

$$
f\left(\Delta \xi_{\max }, P e\right)=-\frac{\Delta \xi_{\max }}{P e}+\frac{2}{P e} .
$$

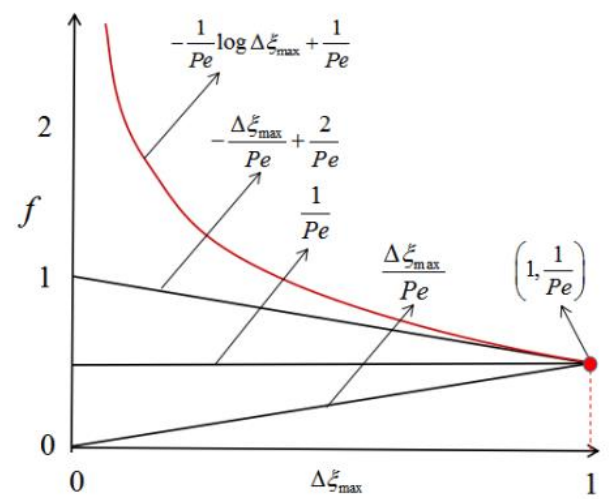

Fig. 1 Different functions for $f\left(\Delta \xi_{\max }, P e\right)$ [28]

For there should be no artificial viscosity in smooth region, the shock indicator is necessary. Persson introduced the shock indicator below. First, the solution polynomial is expressed with orthogonal basis

$$
u=\sum_{i=1}^{N(p)} \widehat{u}_{i} \psi_{i} .
$$

Here choose density as $u_{.} N(p)$ is the number of basis function, $\psi_{i}$ is the orthogonal basis, $\widehat{u}_{i}$ is the 
coefficient for each basis function. And for each element $e$ give the truncated P-1-th order polynomial

$$
\hat{u}=\sum_{i=1}^{N(p-1)} \widehat{u}_{i} \psi_{i} .
$$

And we can get the smooth indicator

$$
\begin{aligned}
& S_{e}=\frac{(u-\hat{u}, u-\hat{u})_{e}}{(u, u)_{e}}, \\
& S_{e}=\log _{10} S_{e}
\end{aligned}
$$

here $S_{e} \square 1 / p^{4}, s_{0}=-3 \log _{10} p, \kappa$ is an experimental coefficient which is basically chosen between 3 and 6.

\subsection{In-cell Piecewise Integrated Solution}

The In-cell Piecewise Integrated solution is a shock capturing method proposed by Huera [30] applied in DG scheme and extended to FR by Yi L U [31]. The idea of In-cell Piecewise Integrated solution is to construct constant solution in each sub-cell and reconstruct the distribution function. It doesn't need neighboring cell's information compared to artificial viscosity method. In order to construct the piecewise constant solution, need to partition each cell into sub-cells containing solution points. For FR scheme, the solution points are located inside cell, the sub-cells could be constructed containing each point. The constant solution at each solution point can be constructed as

$$
u_{e, j}^{\prime}=\frac{1}{V_{j}} \int_{e_{j}} u_{e}(\xi) d e .
$$

Here $e_{j}$ is the sub-cell containing $\mathrm{j}$-th solution point, $V_{j}$ is the volume of $e_{j}$. The new solution $u_{e, j}^{\text {new }}$ at each solution point could be obtained as follow

$$
u_{e, j}^{n e w}=(1-\varepsilon) u_{e, j}+\varepsilon u_{e, j}^{\prime},
$$

here $\varepsilon \in[0,1]$, it depends on the smoothness of solution. And it can be calculated with aforementioned shock indicator.

\subsection{Adaptive mesh refinement method}

In this paper, the AMR library p4est was applied to realize AMR efficiently. P4est [35] is an open source AMR library provides many useful functions. Based on octree data structure, p4est has a complete system of parallel generating mesh, refining mesh, coarsening mesh, load balancing, cell searching, etc. It has a high parallel efficiency and is scalable. And the code is developed under the framework of p4est.

\subsubsection{Grid adaption criteria}

In AMR, besides the complicate data structure and the algorithms, another important question is the decision whether to refine or coarsen the grid during simulation. In fluid dynamics the common choice is the gradient of physical variables, which regards that regions where flow changes rapidly 
have important influence to the precision. And many physical variables could be chosen as indicator like density, temperature, pressure, entropy, etc.

Basically, there are some differences in the choice of indicator for different flows. In flows that contain discontinuities, the shock detector could be used as the indicator. When $s_{e}<s_{0}-\kappa$, which means solution is smooth, the grid is marked "coarsen". Otherwise it indicates that discontinuities exist, and the grid is marked "refine". In flows without discontinuity, the vortex flow is more important. To accomplish a better vortex simulation, the following criteria presented in [36] is used

$$
\tau_{c i}=|\nabla \times \mathbf{V}| d_{i}^{\frac{3}{2}}
$$

$$
\sigma_{c}=\sqrt{\frac{\sum_{i=1}^{N} \tau_{c i}^{2}}{N}}
$$

Where $\boldsymbol{V}$ is the speed vector, $d_{i}=\sqrt{V_{i}}, V_{i}$ is the volume of the i-th cell. $N$ is the number of cells. If $\tau_{c i}>\sigma_{c}$, mark the cell "refine", otherwise mark the cell "coarsen".

\subsubsection{Nonconforming data projection}

As mentioned above, FR scheme needs to calculate the common flux on each cell boundary at each time step. When computational grid is uniform and stationary, the edges of neighboring cells have the same length and flux points are collocated. And common numerical flux can be calculated directly. When AMR is applied, the common edges may be not equal length, called non-conforming edge. In this case, the flux points on each edge are not collocated, which is shown in Fig. 2. To calculate common flux, some projection needs to be conducted. Here the Mortar Element Method (MEM)[37][38] is applied. And the $L 2$ projection is applied to project data when mesh is refined or coarsened.

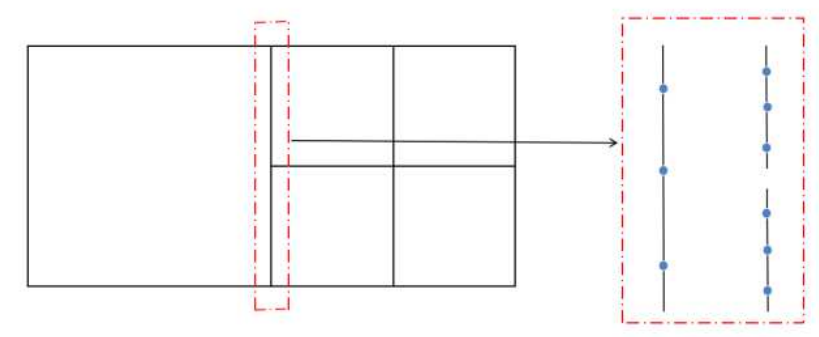

Fig. 2 Nonconforming edge

\section{$4 \quad$ Numerical results}

In order to test the shock capturing ability of FR scheme, we have tested several shock cases in both one and two dimensional. The shock capturing with AMR is also tested in the Euler vortex and double Mach reflection cases. And the time marching method applied here is the three stage RungeKutta method. 


\subsubsection{SOD shock tube}

The SOD shock tube problem is a classic case of shock capturing, with the initial condition

$$
\begin{array}{ll}
\left(\rho_{L}, u_{L}, p_{L}\right)=(1.0,0.0,1.0) & x<0.5 \\
\left(\rho_{R}, u_{R}, p_{R}\right)=(0.125,0.0,0.1) & x \geq 0.5
\end{array} .
$$

In this paper, the grid size is $d x=1 / 100$. Fig. 3 shows the results with artificial viscosity and incell piecewise constant approximation methods. And they are compared with different order at $\mathrm{t}=0.2 \mathrm{~s}$. From the results we can see that both the methods show satisfying results of shock capturing, which solve shock precisely with little oscillation. On the other hand, the numerical solution is more precise with the solution order increased, which is also the same as expected.

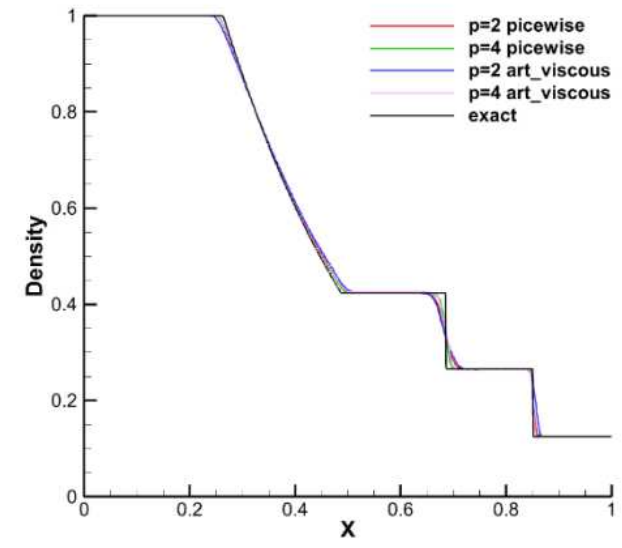

(a) Density distribution

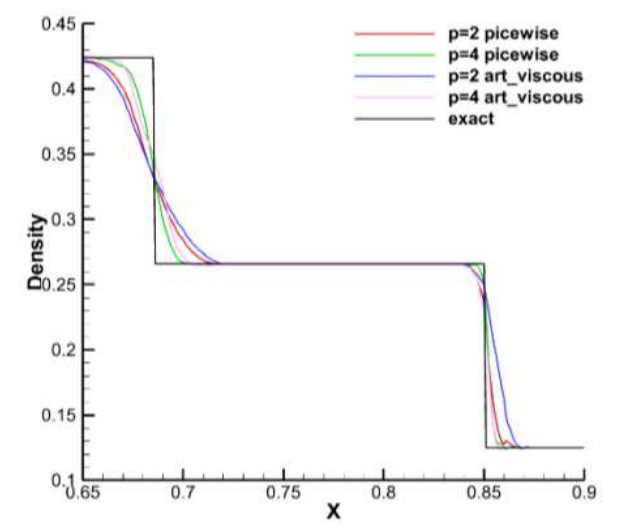

(b) Zoom in result

Fig. 3 Density results of SOD shock tube

\subsubsection{One dimensional Shu-Osher problem}

The well-known Shu-Osher problem is the interaction of a density sine wave with a shock with speed of Mach 3 moving to right. It contains small flow structures and shock. The initial condition of Shu-Osher problem is

$$
\begin{aligned}
& \left(\rho_{L}, u_{L}, p_{L}\right)=(3.857413,2.629369,10.333) \quad x<-4 \\
& \left(\rho_{R}, u_{R}, p_{R}\right)=(1+0.2 \sin (5 x), 0.0,1.0) \quad x \geq-4 \text {. }
\end{aligned}
$$

The computational domain is $[-5,5]$, the grid size is $1 / 20$, the order of accuracy is 3 , Fig. 4 shows the density results at $\mathrm{t}=1.8 \mathrm{~s}$. From the results, we can see that both the methods are capable of capturing small flow structure and there is no obvious oscillation near shock. 


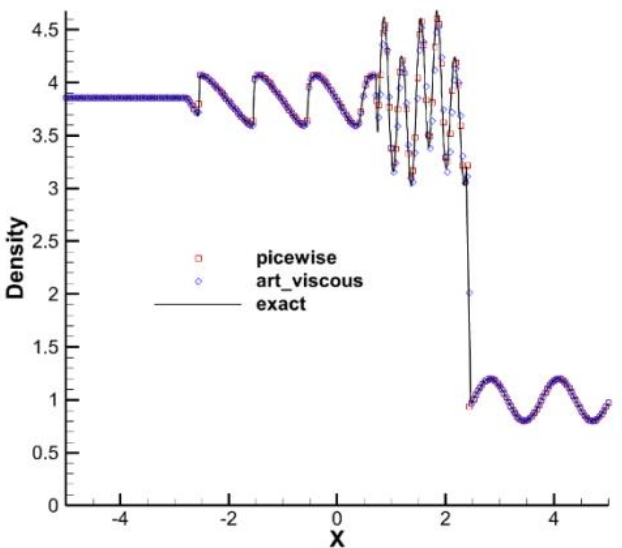

(a) Density distribution

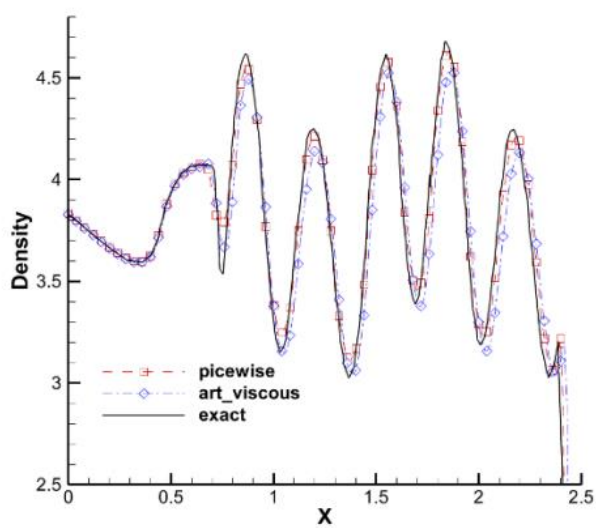

(b) Zoom in result

Fig. 4 Density results of Shu-Osher problem

\subsection{Two dimensional cases}

\section{0}

\subsubsection{Shock-Vortex interaction}

The shock-vortex interaction flow of the stationary shock of Mach 1.1 interacts with an isentropic vortex. Here third order scheme is applied. The case has a computational domain of $[0,2] \times[0,1]$, the grid size is $1 / 60$, the stationary shock is placed at $x=0.5$. Pre-shock initial condition is $\left(\rho_{L}, u_{L}, v_{L}, p_{L}\right)=(1.0,1.1 \sqrt{\gamma}, 0.0,1.0)$, the isentropic vortex center is located at $\left(x_{c}, y_{c}\right)=(0.25,0.25)$, the vortex flow condition on the left of shock is

$$
\begin{aligned}
& \rho=\left(1-\frac{\gamma-1}{4 \alpha \gamma} \varepsilon^{2} e^{2 \alpha\left(1-\tau^{2}\right)}\right)^{\frac{1}{\gamma-1}} \\
& u=\varepsilon \tau e^{\alpha\left(1-\tau^{2}\right)} \cos \theta \\
& v=\varepsilon \tau e^{\alpha\left(1-\tau^{2}\right)} \sin \theta \\
& p=\rho^{\gamma}
\end{aligned}
$$

Here $r=\sqrt{\left(x-x_{c}\right)^{2}+\left(y-y_{c}\right)^{2}}, \varepsilon$ is the vortex strength, $\alpha$ is the decay rate, $r_{c}$ is the radius of vortex, $\theta$ is the angle between the line solution point and vortex center with $\mathrm{x}$ axis. In this paper, $\varepsilon=0.3, \alpha=-0.204, r_{c}=0.05$. Fig. 5 shows the pressure results at $\mathrm{t}=0.8 \mathrm{~s}$ with both artificial viscosity and in-cell piecewise constant approximation.

From this case it can be seen that both the methods can capture shock without affecting the vortex precision. In spite of these results, another results is the artificial viscosity runs slower compared with the in-cell piecewise constant approximation. The problem is although the artificial viscosity could get better results, but it has some problem dependent parameters which are not easy to determine. 


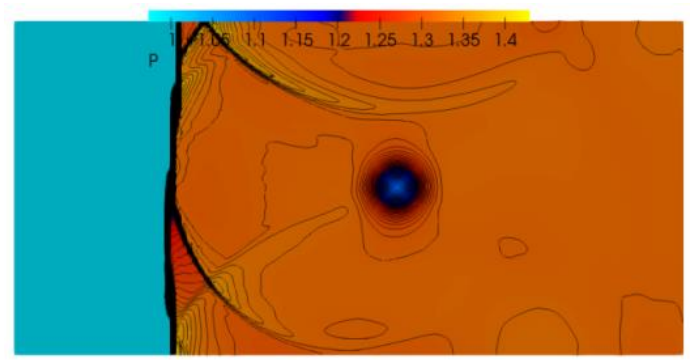

(a) Result with Artificial viscosity

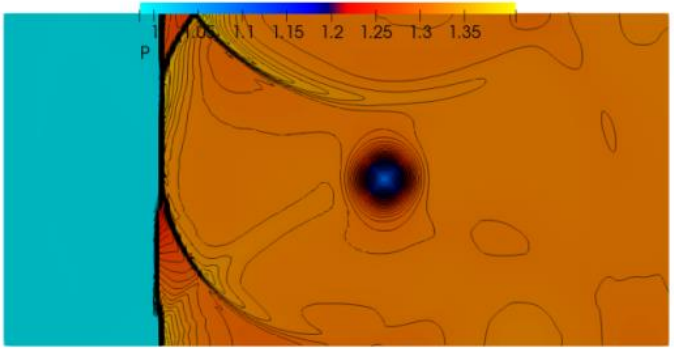

(b) Result with piecewise constant approximation

Fig. 5 Pressure contour results of Shock-vortex Interaction

\subsection{Cases with adaptive mesh refinement}

\section{1}

292

293

\subsubsection{The isentropic Euler vortex}

The isentropic vortex is a classical case usually used to test the precision of numerical schemes. It adds a vortex flow to the uniform flow of initial condition of $\left(\rho_{\infty}, u_{\infty}, v_{\infty}, p_{\infty}\right)=(1,1,1,1),\left(x_{c}, y_{c}\right)$ is vortex center, the initial vortex condition is

$$
\begin{aligned}
& \delta u=-\frac{\varepsilon}{2 \pi}\left(y-y_{c}\right) e^{0.5\left(1-R^{2}\right)} \\
& \delta v=\frac{\varepsilon}{2 \pi}\left(x-x_{c}\right) e^{0.5\left(1-R^{2}\right)} \\
& \delta T=-\frac{(\gamma-1) \varepsilon^{2}}{8 \gamma \pi^{2}} e^{1-R^{2}} \\
& R=\sqrt{\left(x-x_{c}\right)^{2}+\left(y-y_{c}\right)^{2}}
\end{aligned}
$$

Here $\varepsilon=5$, the computational domain is $0 \leq x \leq 10,0 \leq y \leq 10$, the vortex center is located at $(5,5)$, the boundary condition is periodic condition, and the grid is $20 \times 20$. In this paper, the FR scheme with AMR is tested with this case. Here give the results at $\mathrm{t}=10 \mathrm{~s}$. Fig. 6 shows the computational grid at different time. Fig. 7 shows the density distribution with different order and with AMR. From these results we can see that the grid could automatically refine and coarsen with the vortex indicator without affecting precision, which greatly improves the simulation.
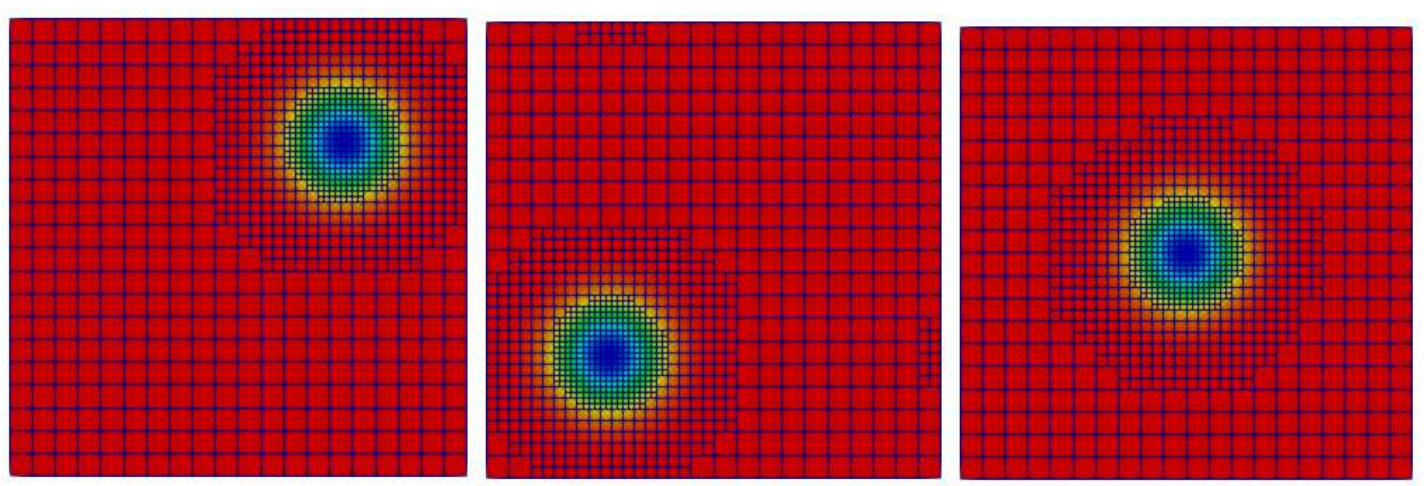

Fig. 6 Mesh distribution of Euler vortex at different time 

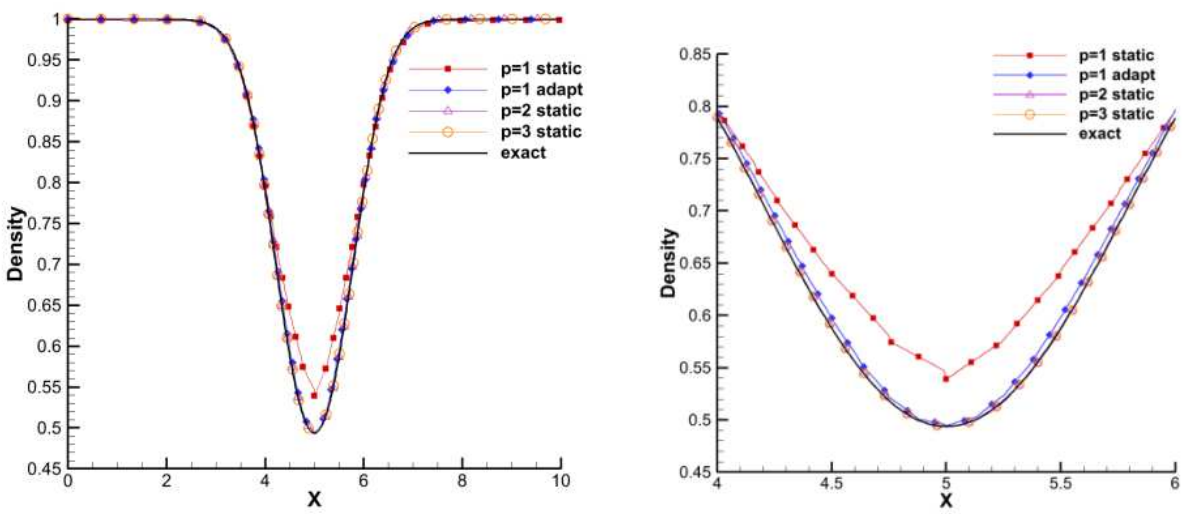

Fig. 7 Density distribution at $y=0$

\subsubsection{Oblique shock reflection}

In this paper, mainly third order scheme is applied for 2D cases. First we tested the oblique shock reflection problem. The initial condition is the oblique shock of Mach 3 reflected on an inviscid wall with an inflow angle of 29 degree respect to $\mathrm{x}$ axis. The results are shown in Fig. 9, it's easy to find out that with AMR the shock is much sharper than the original one.

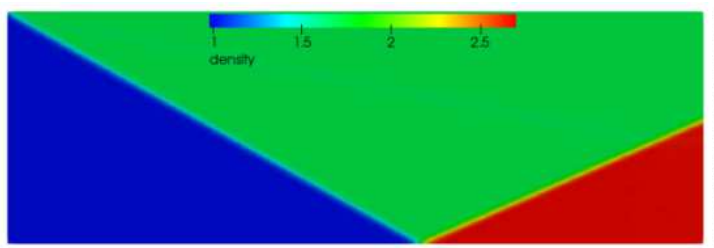

(a) Density result

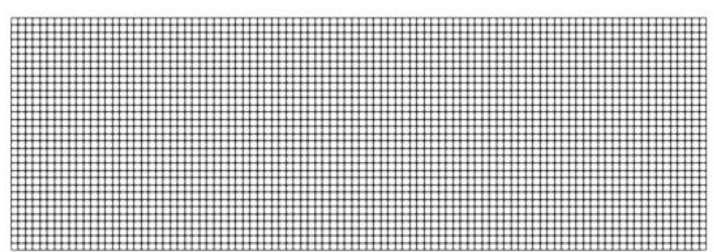

(b) Mesh Distribution

Fig. 8 Results of oblique shock reflection without AMR

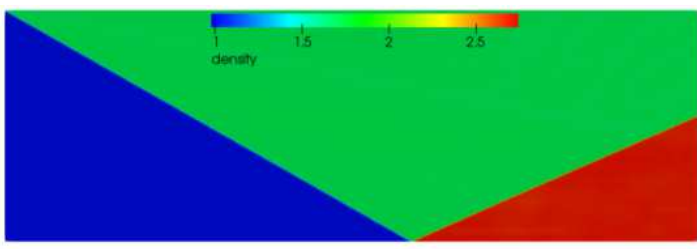

(a) Density result

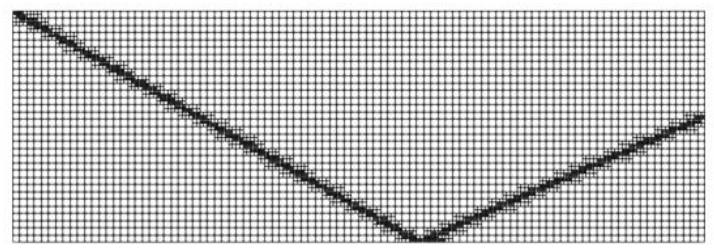

(b) Mesh Distribution

Fig. 9 Results of oblique shock reflection with AMR

\subsubsection{Double Mach reflection}

The double Mach reflection problem is to simulate the flow of an oblique shock of Mach 10 is used to test the scheme's ability of capturing strong shock. The double Mach reflection problem has a computational domain of $[0,4] \times[0,1]$, the lower boundary at $0 \leq x<1 / 6$ and the left boundary are set the post-shock initial condition, lower boundary at $1 / 6 \leq x \leq 4$ is set the reflect wall condition., right boundary is set free outflow condition, upper boundary is set the same as the exact movement of shock. The pre-shock and post-shock initial condition is

$$
\begin{aligned}
& \left(\rho_{\text {pre }}, u_{\text {pre }}, v_{\text {pre }}, p_{\text {pre }}\right)=(1.4,0,0,1) \\
& \left(\rho_{\text {post }}, u_{\text {post }}, v_{\text {post }}, p_{\text {post }}\right)=\left(8,8.25 \cos 30^{\circ},-8.25 \sin 30^{\circ}, 116.5\right)
\end{aligned}
$$


In this case, the initial grid size is $1 / 60$, with third order simulation, and capture shock with artificial viscosity. Fig. 10 shows the density contour and grid distribution at $t=0.2 \mathrm{~s}$. Fig. 11 shows the zoom in result of Double Mach Reflection. From these results, we can see that with artificial viscosity, the shock could be captured precisely, and with the grid refinement in shock region, the shock is sharp and flow structure is precise.

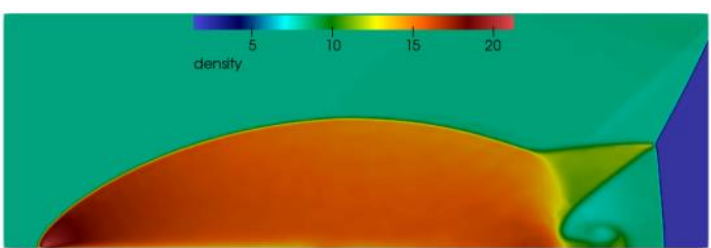

(a) Density result

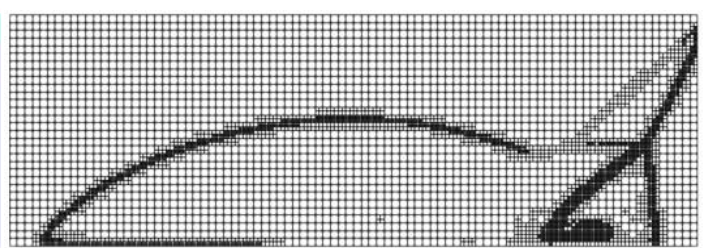

(b) Mesh Distribution

Fig. 10 Results of Double Mach Reflection

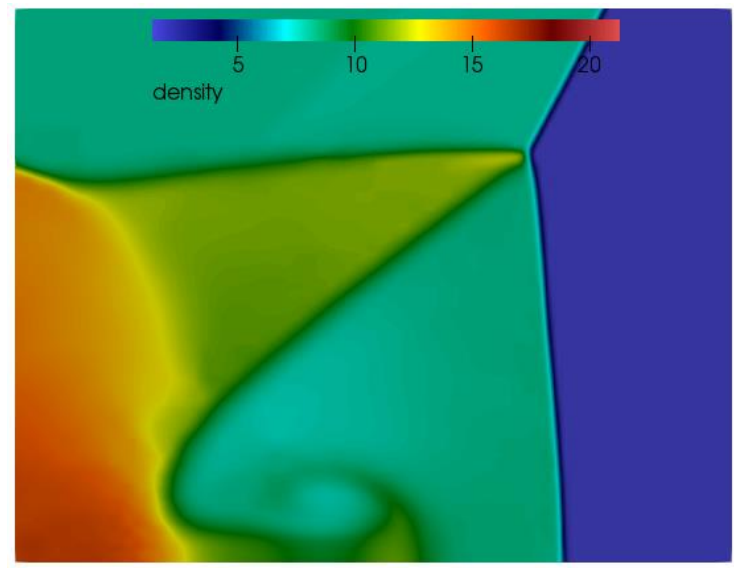

\section{Summary}

In this paper, FR scheme is realized based on the framework of AMR library p4est in two dimensional. Compare the performance of shock capturing ability of the Artificial Viscosity and Incell Piecewise Integrated solution methods. Both of them show pleasant results, the Artificial Viscosity can have more precise results but is more complex and needs to adjust parameters which are problem dependent.

With the help of p4est, the AMR is added to the code. From the simulation of both subsonic and supersonic cases we can see that the AMR is successfully applied to FR scheme.

\subsection{Further work}

1. Only cases in Euler equations are simulated, the Navier-Stokes equations will be added to the code;

2. Three dimensional simulation will be added to the system;

3. The curved boundary needs to be applied in real geometry;

4. The time marching method is Runge-Kutta method, which has a strict limitation to time step, later the implicit time marching methods would be added. 
356 The authors would like to acknowledge for the sponsorship of National Numerical Windtunnel project.

\section{Authors' contributions}

XQM is mainly responsible for the coding of Flux Reconstruction Method and data analysis. JX designed the framework of the program.

This work is supported by National Numerical Windtunnel project.

362

\section{Availability of data and materials}

All data generated or analysed during this study are included in this published article

\section{Competing Interests}

The authors declare that they have no competing interests.

\section{Author details}

*Correspondence: jxia@nuaa.edu.cn

1 College of Aerospace Engineering, Nanjing University of Aeronautics and Astronautics, Nanjing, 210016, China

\section{References}

1. Reed, W.H, Hill, T.R (1973) Triangular Mesh Methods for the Neutron Transport Equation. Los Alamos Report La, 1973.

2. B. Cockburn, C.-W. Shu (1989) TVB Runge-Kutta local projection discontinuous Galerkin finite element method for conservation laws II: general framework, Math. Comput. 52 (186): 411-435. https://doi.org/10.2307/2008474

3. Kopriva, D. A., Kolias, J. H (1996). A conservative staggered-grid chebyshev multidomain method for compressible flows. J Comput Phys 125(1): 244-261. https://doi.org/10.1006/jcph.1996.0225

4. WANG, Z. J (2004) Spectral (finite) volume method for conservation laws on unstructured grids: Basic formulation. J Comput Phys 194(2): 716-741. https://doi.org/10.1006/jcph.2002.7041

5. Yen Liu, Marcel Vinokur, Z J Wang (2006). Spectral difference method for unstructured grids I: Basic formulation. J Comput Phys 216 (2): 780-801. https://doi.org/10.1016/j.jcp.2006.01.024

6. Huynh, H.T.. (2007). A Flux Reconstruction Approach to High-Order Schemes Including Discontinuous Galerkin Methods. AlAA Paper 2007-4079. https://doi.org/10.2514/6.2007-4079

7. Wang Z J, Gao H (2009) A unifying lifting collocation penalty formulation including the discontinuous Galerkin, spectral volume/difference methods for conservation laws on mixed grids. J Comput Phys 228(21):8161-8186. https://doi.org/10.1016/j.jcp.2009.07.036

8. Gao H, Wang Z J (2009). A high-order lifting collocation penalty formulation for the Navier-Stokes equations on 2-D mixed grids. AIAA-2009-3784 . https://doi.org/10.2514/6.2009-3784

9. Gao H, Wang Z J, Huynh HT (2013). Differential formulation of discontinuous Galerkin and related methods for the Navier-Stokes equations. Commun Comput Phys 13(4):1013-44. https://doi.org/10.4208/cicp.020611.090312a

10. Haga T, Gao H, Wang Z J (2010) A high-order unifying discontinuous formulation for 3-D mixed grids. AIAA Paper 2010-540. https://doi.org/10.2514/6.2010-540

11. Jameson, A (2010) A proof of the stability of the spectral difference method for all orders of accuracy. J Sci Comput 45(1-3): 348358. https://doi.org/10.1007/s10915-009-9339-4

12. Vincent P E, Castonguay P, Jameson A (2011) A New Class of High-Order Energy Stable Flux Reconstruction Schemes. J Sci Comput 47(1):50-72. http://dx.doi.org/10.1007/s10915-010-9420-z

13. Kartikey Asthana, Antony Jameson (2015) High-Order Flux Reconstruction Schemes with Minimal Dispersion and Dissipation. Plenum Press. http://doi.org/10.1007/s10915-014-9882-5 
14. Romero J , Asthana K, Jameson A (2016) A Simplified Formulation of the Flux Reconstruction Method. J Sci Comput 67(1):351-374. http://doi.org/10.1007/s10915-015-0085-5

15. Joshua Romero, Antony Jameson(2015) Extension of the Flux Reconstruction Method to Triangular Elements using Collapsed-Edge Quadrilaterals. Aiaa Scitech Aiaa Aerospace Sciences Meeting. http://doi.org/10.2514/6.2016-1825

16. Romero J , Witherden F D , Jameson A (2017). A Direct Flux Reconstruction Scheme for Advection-Diffusion Problems on Triangular Grids. J Sci Comput 73(2-3):1115-1144 http://doi.org/10.1007/s10915-017-0472-1

17. Wang, L., Yu, $\mathrm{M}(2018)$ Compact Direct Flux Reconstruction for Conservation Laws. J Sci Comput 75: 253-275 . https://doi.org/10.1007/s10915-017-0535-3

18. Cockburn, Bernardo, and Chi-Wang Shu.(1989) TVB Runge-Kutta local projection discontinuous Galerkin finite cell method for conservation laws. II. General framework. Math Compute 52(186): 411-435. https://doi.org/10.2307/2008474

19. Du J, Shu CW , Zhang M (2015) A simple weighted essentially non-oscillatory limiter for the correction procedure via reconstruction (CPR) framework on unstructured meshes. Appl Numer Math 90:146-167. https://doi.org/10.1016/j.apnum.2014.12.004

20. Jin Seok Park, Tae Chang, Chongam Kim. (2014). Higher-order Multi-dimensional Limiting Strategy for Correction Procedure via Reconstruction. 52nd AIAA Aerospace Sciences Meeting - AIAA Science and Technology Forum and Exposition, SciTech 2014. https://doi.org/10.2514/6.2014-0772.

21. Christopher Michalak, Carl Ollivier-Gooch(2009) Accuracy preserving limiter for the high-order accurate solution of the Euler equations. J Sci Comput 228 (23) 8693-8711. https://doi.org/10.1016/j.jcp.2009.08.021

22. Li Yanan, Wang Z. J. (2017). A convergent and accuracy preserving limiter for the FR/CPR method. AlAA 2017-0756. https://doi.org/10.2514/6.2017-0756

23. Cook, Andrew W (2007). Artificial fluid properties for large-eddy simulation of compressible turbulent mixing. Phys Fluids 19(5):055103. https://doi.org/10.1063/1.2728937

24. Persson, Per-Olof , Peraire J (2006). Sub-Cell Shock Capturing for Discontinuous Galerkin Methods. AIAA paper. 2006-112 . https://doi.org/10.2514/6.2006-112

25. Kawai S, Lele S K (2008) Localized artificial diffusivity scheme for discontinuity capturing on curvilinear meshes. J Comput Phys, 227(22):9498-9526. https://doi.org/10.1016/j.jcp.2008.06.034

26. Yu M L, Giraldo F X , Peng M, et al(2015). Localized Artificial Viscosity Stabilization of Discontinuous Galerkin Methods for Nonhydrostatic Mesoscale Atmospheric Modeling. Mon Weather Rev. https://doi.org/10.1175/MWR-D-15-0134.1

27. Premasuthan S, Liang C , Jameson A (2014) Computation of flows with shocks using the Spectral Difference method with artificial viscosity, I: Basic formulation and application. Comput Fluids 98:111-121. https://doi.org/10.1016/j.compfluid.2013.12.013

28. Yu M , Wang Z J (2014) Shock Capturing for Correction Procedure via Reconstruction Methods Using Artificial Viscosity and Diffusivity. Eighth International Conference on Computational Fluid Dynamics. 2014.

29. Trojak W, Watson R , Tucker P (2018). Temporal Stabilisation of Flux Reconstruction on Linear Problems. https://doi.org/10.2514/6.2018-4263

30. Huerta A, Casoni E , Peraire J(2012) A simple shock-capturing technique for high-order discontinuous Galerkin methods. Int J Numer Methods Fluids 69(08):1614-1632. https://doi.org/10.1002/fld.2654

31. Yi L U , Liu K, Dawes W N(2015) Flow simulation system based on high order space-time extension of flux reconstruction method. 53rd AIAA Aerospace Sciences Meeting. https://doi.org/10.2514/6.2015-0833

32. Shephard, M (1985) Automatic and adaptive mesh generation. IEEE Trans Magn 21(6):2484-2489. https://doi.org/10.1109/tmag.1985.1064241

33. Zhang S, Liu,J J, Chen Y S, Wang T S. (2001). Adaptation for hybrid unstructured grid with hanging node method. 15th AlAA Computational Fluid Dynamics Conference. https://doi.org/10.2514/6.2001-2657

34. Jameson A, Vincent P E , Castonguay P (2012) On the Non-linear Stability of Flux Reconstruction Schemes. J Sci Comput 50(2):434445. https://doi.org/10.1007/s10915-011-9490-6.

35. Burstedde C, Wilcox L C, Ghattas O (2011) p4est : Scalable Algorithms for Parallel Adaptive Mesh Refinement on Forests of Octrees. 
441 36. Darren D, Kenneth G. Powell(1992) Euler calculations of axisymmetric under-expanded jets by an adaptive-refinement method. Aerospace Sciences Meeting \& Exhibit. https://doi.org/10.2514/6.1992-321

443

37. Zhang B, Liang C(2015). A simple, efficient, and high-order accurate curved sliding-mesh interface approach to spectral difference method on coupled rotating and stationary domains. J Comput Phys, 295:147-160. https://doi.org/10.1016/j.jcp.2015.04.006 